\section{Periodontal Regeneration: Promising and Challenging for Periodontal Complex Regeneration}

Received: March 23, 2017; Accepted: March 24, 2017; Published: March 27, 2017

\section{Shue Li \\ Department of Stomatology, Wuhan Union Hospital, China}

Corresponding author: Shue Li

lishue737@hotmail.com Department of Stomatology, Wuhan Union
Hospital, China.

Tel: $13437295043,+86-027-85726165$

Citation: Li S. Periodontal Regeneration: Promising and Challenging for Periodontal Complex Regeneration. J Bone Rep Recomm. 2017, 3:1.

Derivative (EMD) and Platelet-Derived Growth Factor (PDGF), insulin-like growth factor-I and Bone Morphogenetic Proteins (BMPs) have been introduced in researches with excellent results. However, the clinical significance of these procedures is still to be seen.

For stem cell engineering, the main cell populations with regard to periodontal regeneration, including Bone Marrow-Derived Mesenchymal Stem Cells (BMSCs) and the main dental-derived mesenchymal stem cell populations: Periodontal Ligament Stem Cells (PDLCs), Dental Pulp Stem Cells (DPSCs), Stem Cells from Human Exfoliated Deciduous Teeth (SHED), Stem Cells from Apical Papilla (SCAP) and Dental Follicle Precursor Cells (DFCs) and MSCs from gingival tissues. Cell injection, cell sheet pellets or micro tissues for tissue regeneration has the potential to significantly influence periodontal treatment strategies in the future. Despite the promising future, there are still many issues that need to be dealt. For example, difficulties in trigger of the differentiation of cell, large-scale expansion of cell, potential risk of transmitting vial and prion disease with the use of Fetal Bovine Serum (FBS).

Regeneration of bone-PDL complexes is promising but challenging due to complexes topologies and tissue integration required for functional restoration. Other clinical factors are also included in final. Outcome firstly, defect dimensions appear to be an important factor in predicting regeneration outcomes in clinic. It's found that wide shallow defects responded with less bone gain compared with narrow deep intrabony defects, in which better clinical results are achieved (i.e., larger clinical attachment level gain and bone fill). Second, the amount and character of residual 
gingiva is critical for achieving passive flap adaptation and wound closure for primary intention healing. Given full soft coverage and appropriate thickness of gingiva coverage, the healing process could not be interrupted. However, it's also indicated time is an important factor. Given enough time for tissue remodelling and maturation, even very large periodontal defects grafted with a bone substitute by guided tissue regeneration showed complete regeneration. Above all, many advances have been made over the past few decades for the regeneration of periodontal apparatus. Tissue engineering has been developed from the application of one type of material to combinations of different biomaterials and finally to a delivery system to biological factors and cells. More research needs to be focused on in vivo systems or randomized clinical researches to improve the results. 